\title{
Impacts de la Décharge Publique d'Akouédo sur le Cadre de Vie et la Santé de la Population Riveraine
}

\section{Henri-Michel Obe,}

Titulaire d'un Master en Droit Politique et Gestion de l'Environnement, Université Méthodiste, Abidjan, Côte d'Ivoire

\section{Dr. Brou Ahossi Nicolas,}

Sociologue de l'Environnement, Enseignant-Chercheur, Université Félix Houphouët-Boigny, Abidjan, Côte d'Ivoire

Doi: 10.19044/esj.2019.v15n12p20 ＵRL:http://dx.doi.org/10.19044/esj.2019.v15n12p20

\section{Résumé}

La décharge publique d'Akouédo constitue une préoccupation majeure tant pour les autorités du District d'Abidjan, responsables de la gestion des déchets ménagers, que pour les riverains. L'objectif de cet article est de mettre en exergue l'impact que peut produire une décharge non contrôlée sur le cadre de vie et le bien-être de la population riveraine. Pour mener à bien cette étude, nous avons adopté deux démarches complémentaires. D'une part, à partir d'une démarche qualitative, nous avons mené des entretiens semi-directifs sur le site de la décharge d'Akouédo. Cette approche nous a permis de connaître cette décharge publique, les sociétés qui l'exploitent et surtout d'observer les différentes étapes de la gestion des déchets ménagers solides dans ladite décharge. D'autre part, à partir d'une démarche quantitative, nous n'avons pas hésité à approcher la population riveraine pour mieux appréhender les impacts environnementaux et sanitaires, eu égard au problème complexe que pose la saturation de la décharge d'Akouédo.

Mots-clés : Akouédo, décharge publique, déchets ménagers, environnement, gestion 


\title{
The Impact of the Akouedo Landfill on the Living Environment and the Health of the Local Population
}

\author{
Henri-Michel Obe, \\ Titulaire d'un Master en Droit Politique et Gestion de l'Environnement, \\ Université Méthodiste, Abidjan, Côte d'Ivoire \\ Dr. Brou Ahossi Nicolas, \\ Sociologue de l'Environnement, Enseignant-Chercheur, Université Félix \\ Houphouët-Boigny, Abidjan, Côte d'Ivoire
}

\begin{abstract}
The Akouedo public wasteland is a major concern for the authorities of the District of Abidjan, who are responsible for the management of household waste and the local population. This paper focuses on highlighting the impact that an uncontrolled public wasteland can have on the living environment and the well-being of the local population. In carrying out this study, we adopted two complementary approaches. Based on a qualitative approach, we conducted semi-directive interviews on the Akouedo public wasteland site. That approach allowed us to know the Akouedo public wasteland, the operating companies, and above all to observe the different stages of solid household waste management in that public wasteland. Based on a quantitative approach, we did not hesitate to approach the local population to well understand the environmental and health impacts, having regards for the complex problem posed by the saturation of the Akouedo public wasteland.
\end{abstract}

Keywords: Akouedo, public wasteland, household waste, environment, management

\section{Introduction}

L'Afrique connait une urbanisation rapide, processus nourri par une croissance démographique élevée. Cette évolution de la population, exceptionnelle par son ampleur et sa rapidité, s'accompagne de dynamiques spatiales nouvelles, tant en termes de migration de population que d'urbanisation (Sanogo, 2015).

Cette croissance du nombre d'habitants a aussi pour corollaire la production de quantités très importantes d'ordures ménagères. Ce constat fait de ces ordures un sujet d'actualité récurrent dans nos états africains toujours 
impuissants dans la quête d'une solution durable. En effet, une gestion partielle et inadéquate des ordures ménagères a des conséquences néfastes sur notre environnement (la pollution des sols, de l'eau et de l'air), et, par ricochet sur la santé, les activités et les biens et des usagers (Hebette, 1996). Nous assistons à un système de traitement linéaire des déchets ; c'est-à-dire, un traitement qui se limite aux opérations de collecte, de transport et de mise en décharge dans nos villes africaines.

Abidjan, capitale économique de la Côte d'Ivoire, reste l'une des villes d'Afrique de l'Ouest qui n'échappe guère à ce phénomène de production de quantités très importantes d'ordures ménagères. Cette ville, depuis 1965, s'est dotée d'une décharge à ciel ouvert destinée à traiter et à éliminer dans des conditions plus ou moins réglementées tous les déchets (Adjiri, 2015). Cette décharge est située à $16 \mathrm{~km}$ du centre-ville et à $0,6 \mathrm{~km}$ au sud de la route nationale 105 sur l'axe Abidjan - Bingerville. Elle est localisée dans la commune de Cocody à proximité du village Ebrié «Akouédo » dont elle porte le nom (voir Figure 1).

Les gouvernements successifs, depuis la création de ce dépôt d'ordures jusqu'aujourd'hui, soucieux du cadre de vie et du bien-être des citadins, se sont dotés de services urbains pour promouvoir leurs politiques respectives de salubrité urbaine. Malheureusement, la gestion de la décharge d'Akouédo a montré ses limites dans le temps. Ce dépotoir non contrôlé recevait des quantités très importantes de déchets urbains (ménagers, biomédicaux, industriels dangereux) jusqu'à sa fermeture en novembre 2018. Les pratiques de traitement et d'élimination des déchets dans ledit dépotoir sont néfastes tant pour le milieu naturel que pour la santé de la population riveraine.

À cet égard, la présente réflexion a pour objectif global de montrer les répercussions résultant des pratiques des opérateurs techniques dans la gestion des déchets urbains. Plus spécifiquement, l'étude vise à apprécier les moyens et techniques dont disposent les opérateurs pour le traitement et l'élimination des déchets urbains entreposés à la décharge publique d'Akouédo. Pour ce faire, la méthodologie, ainsi que les résultats et discussions qui ont meublé cette étude seront présentés ci-après.

\section{Méthodologie}

\section{I.1.Lieu de l'étude}

La décharge publique d'Akouédo est une décharge non contrôlée située près du village d'Akouédo. Elle est limitée au nord et à l'ouest par les quartiers résidentiels de la Riviera, à l'est par le village Akouédo-Attié et au sud par la baie de M'Badon qui s'ouvre sur la lagune Ébrié et qui constitue la principale limite naturelle. Ce dépôt d'ordures est constitué par un ravin naturel qui s'étend du nord au sud, sur 396000 m à $397500 \mathrm{~m}$ en abscisse et $588000 \mathrm{~m}$ à $592500 \mathrm{~m}$ en ordonnées, dans le référentiel UTM (Universal 
Transverse Mercator), fuseau 30N. Elle s'inscrit dans un sous bassin versant d'une superficie d'environ $70 \mathrm{~km}^{2}$ (Adjiri, 2015).

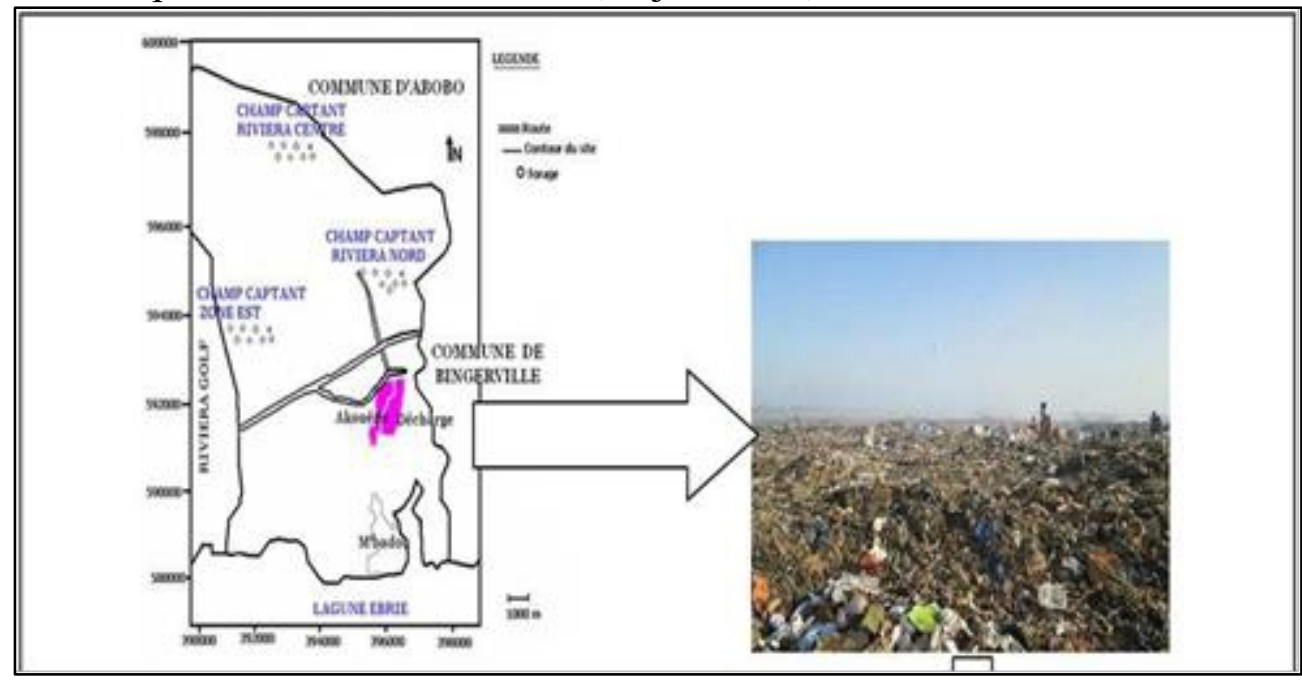

Figure 1. Localisation de la décharge d'Akouédo

Source : BLE, et al. (2014). Évaluation de la qualité physico-chimique des eaux souterraines dans la zone d'Akouédo (Sud de la Côte d'Ivoire).

\section{I .2. Collecte de données}

Les résultats de l'étude ont été obtenus à partir d'un travail de recherche sur le site du dépotoir qui a exigé plusieurs mois de visite (effectuée au cours de la période allant du 11/11/2017 au 30/10/2018). Cette visite a été faite sous la conduite du superviseur du quai de déversement de l'opérateur PFO (Pierre Fakhoury Operator) AFRICA, entreprise ayant en charge l'exploitation de la décharge publique d'Akouédo.

En ce qui concerne l'approche de la population riveraine, l'enquête de terrain a utilisé comme technique en matière d'échantillon, la technique aléatoire, fondée sur le principe du hasard. Ce procédé a permis de choisir au hasard des groupes d'individus existants naturellement dans la réalité. Ainsi avons-nous interrogé 300 ménages des quartiers environnants les plus atteints par le traitement et l'élimination des ordures ménagères stockées. Ce sont les cités Synacassci, Riviera Faya, Génie 2000 et le village d'Akouédo. À côté de ces ménages sujets de l'enquête, nous avons interrogé les opérateurs responsables de l'exploitation du site, les autorités du village d'Akouédo et les acteurs informels (récupérateurs, fouineurs et vendeurs).

\section{I.3. Analyse de données}

Les fiches d'enquêtes ont été dépouillées à l'aide des logiciels « Google Forms » et «Excel» en ce qui concerne l'enquête quantitative. Dans la première étape, il était question de l'utilisation du logiciel « Google Forms ». 
Dans la seconde étape, à l'aide du logiciel « Excel», nous avons présenté les résultats obtenus sous forme de secteurs et d'histogramme.

En ce qui concerne l'enquête qualitative, les entretiens semi-directifs ont été transcrits.

\section{Résultats}

\section{II.1. Description et fonctionnement de la décharge d'Akouédo}

La décharge d'Akouédo est une décharge non contrôlée où les ordures sont déversées sans être recouvertes de matériaux inertes. Les ordures ménagères sont entreposées au fur et à mesure du remplissage du ravin puis étalées à l'aide d'engins. Ce dépôt d'ordures dispose d'un poste de pesage où le poids de toutes les ordures collectées est enregistré et d'un pont-bascule installé juste devant cette cabine pour tarifer les camions au volume.

En ce qui concerne les aménagements du site, la voie d'accès à la décharge n'est plus bitumée. Celles qui ont accès aux différents quais sont en terre battue bien drainées. Le système de canalisation le long des voies bitumées existe pour le drainage des eaux pluviales. Il y a aussi des lacs artificiels pour réduire la teneur en contamination des lixiviats avant leur évacuation vers la lagune Ébrié. La clôture de la décharge se confond aujourd'hui avec les habitations mêmes.

\section{II.2. Entreprises exploitantes de la décharge publique d'Akouédo}

Nous avons au plan technique :

- Pierre Fakhoury Operator (PFO) AFRICA, qui est l'entreprise exploitante de la décharge publique d'Akouédo depuis novembre 2017 après une convention signée avec l'Agence Nationale de Salubrité Urbaine (ANASUR) devenue ANAGED ${ }^{1}$ depuis 2018.

- AfricWaste, entreprise qui intervient dans le domaine du recyclage des déchets ménagers solides, précisément les déchets plastiques PET (Polyéthylène-téréphtalate), qui sont plus spécifiquement les bouteilles plastiques d'eau minérale et de boisson gazeuse. À cette entreprise a lieu la troisième étape du traitement des déchets (la valorisation) où ils récupèrent, traitent et éliminent les déchets ménagers solides.

\section{Étapes de traitement et d'élimination des déchets urbains acheminés à la décharge publique d'Akouédo}

Le processus de traitement et l'élimination des déchets urbains commencent au pont-bascule de la décharge d'Akouédo où les contrôleurs vérifient les feuilles de route des camions à benne (provenance du véhicule

\footnotetext{
${ }^{1}$ Agence Nationale de Gestion des Déchets de Côte d'Ivoire
} 
de collecte) ou autre engin transportant les déchets et leur autorisation à déverser les ordures à la décharge publique d'Akouédo. Sur le pont-bascule, les véhicules de collecte sont donc identifiés et pesés en entrée et en sortie pour déterminer le tonnage des déchets entrant à traiter. Les contrôleurs de zone dirigent ensuite les camions à bennes au quai et les orientent pour le déversement dans les ravins en fonction du type de déchet transporté. Les ordures ménagères sont déversées directement sur un quai où la pelle à grappin et les chargeurs les repoussent pour remplir au fur et à mesure le ravin. Ensuite, lorsque les déchets atteignent un certain niveau, ils sont compactés par des compacteurs à pied sans être recouverts de matériaux inertes. Cette première étape est appelée le recouvrement.

L'étape suivante consiste à bruler les déchets à ciel ouvert sur la terre ferme. Pour ce faire, un espace dédié uniquement à cette pratique a été aménagé à la décharge d'Akouédo. Cette deuxième étape est appelée l'incinération.

\section{II.3. Résultat de l'enquête d'opinion auprès de la population riveraine II.3.1. Responsabilité de la décharge publique d'Akouédo}

La figure ci-dessous nous donne une perception des ménages de tous les acteurs concernés dans l'exploitation de cette décharge (Figure 2).

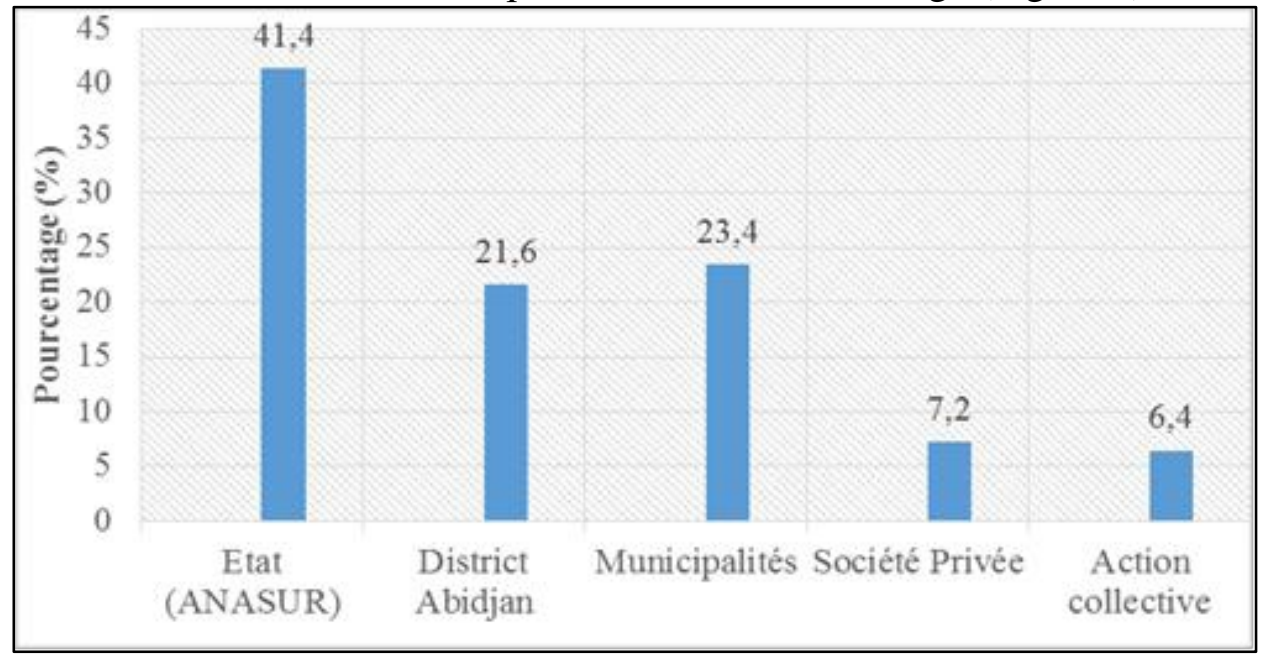

Figure 2. Acteurs en charge de la décharge publique d'Akouédo

Source : (notre étude, 2018)

\section{II.3.2. Appréciation de la gestion quotidienne des déchets urbains par les opérateurs techniques}

La figure ci-dessous nous donne les réels motifs des réactions des ménages en ce qui concerne le traitement et l'élimination des déchets urbains de la décharge publique d'Akouédo (Figure 3). 


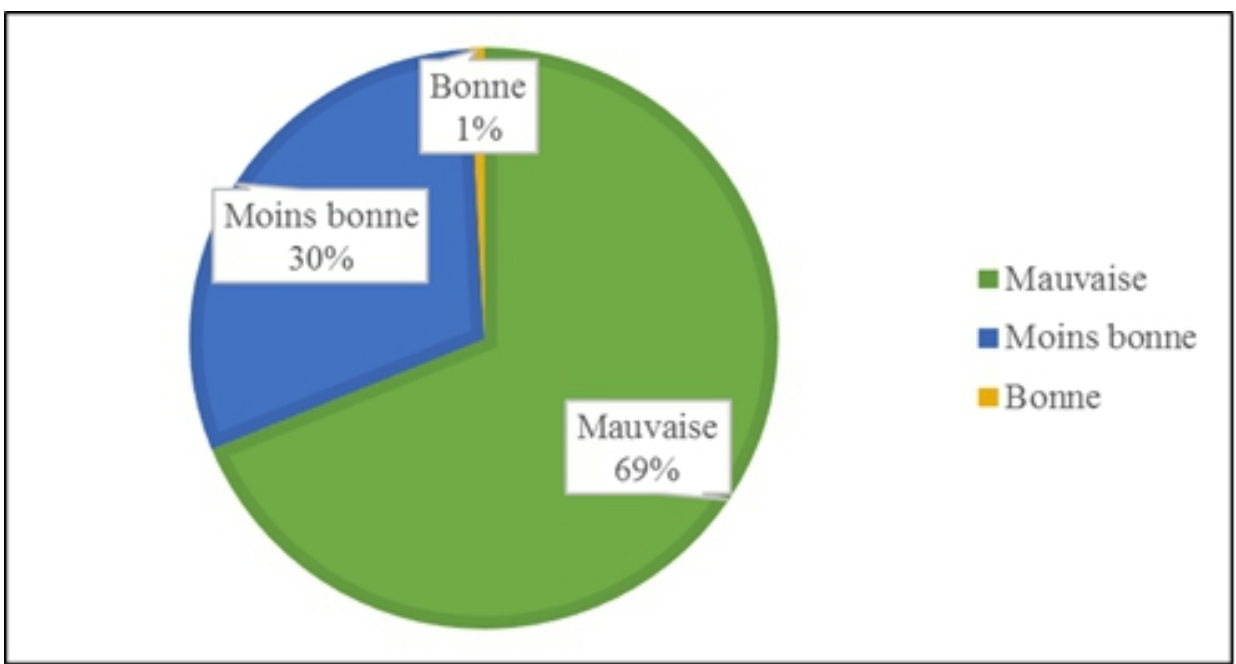

Figure 3. Appréciation de la gestion des déchets urbains sur le cadre de vie Source : (notre étude, 2018)

\section{II.3.3. Effets néfastes de la gestion des déchets urbains}

La figure ci-dessous montre les conséquences de la gestion inadéquate des déchets urbains de la décharge sur la population abidjanaise en général et riveraine en particulier (Figure 4).

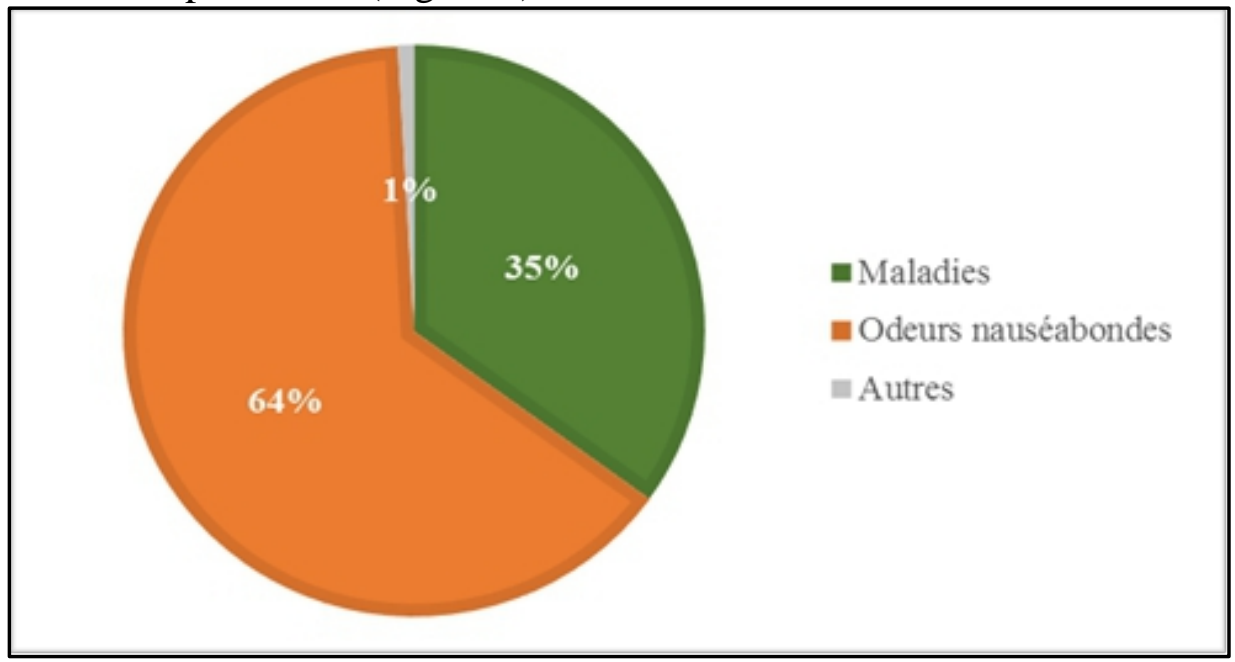

Figure 4. Nuisances produites par l'état de la décharge publique Source : (notre étude, 2018)

Les résultats de l'étude montrent que la population riveraine est fortement impactée par l'état de la décharge publique d'Akouédo qui nuit à son cadre de vie et à sa santé. En effet, cette population est exposée à des odeurs nauséabondes, à des maladies et des symptômes associés à la composition microbiologique des déchets urbains (diarrhée aigüe, fièvre 
typhoïde, infections pulmonaires, etc.) émanant de ce dépotoir. Aussi, on note la présence de moustiques et rongeurs, insectes, mouches qui font la navette entre ce dépôt d'ordures et les lieux d'habitations de ces riverains.

\section{Discussion}

Au cours de l'enquête de terrain, nous avons été souvent confrontés à des restrictions quant à l'accès à certains endroits de la décharge d'Akouédo. Cependant, nos résultats mettent en évidence la nécessité d'une étude de prévalence environnementale et sanitaire régulière dans le voisinage de ce dépôt d'ordures. Et ce, même après sa fermeture définitive.

Cette décharge partage avec bien d'autres en Afrique l'impact négatif qu'elles ont sur les populations habitant près d'elles. D'ailleurs, Lamchouri (2018) confirme les impacts négatifs de l'exploitation confuse et non appropriée de la décharge publique de la ville de Taza (Maroc) qui touchent principalement la santé du personnel de la décharge, des chiffonniers et leurs familles installées près du dépotoir et la santé des habitants des quartiers limitrophes touchés par les odeurs émanant de la décharge. Son étude remet donc en cause les moyens et techniques dont disposent les opérateurs pour le traitement des déchets et le traitement des lixiviats sur les décharges publiques non contrôlées. À vrai dire, la mise en décharge non contrôlée ou l'incinération à ciel ouvert comme moyen d'élimination des déchets urbains dans les pays en voie de développement résulteraient du manque de moyens techniques des opérateurs (Issihaka, 2015). Par ailleurs, un aspect intéressant qu'il soulève est la nécessité d'une étude d'impact environnemental et social (EIES) préalable à la réhabilitation d'un site de stockage des déchets solides ou pollués. Et ce, en respectant à tout moment les réglementations et les législations en vigueur. Cette idée est soutenue par Issihaka (2015) qui met l'accent sur le contrôle régulier des décharges non contrôlées par une équipe compétente de suivi et d'évaluation. Enfin, cette étude d'impact environnemental et social pourrait être appliquée à la décharge non contrôlée d'Akouédo en vue de sa reconversion. $^{2}$

En outre, l'environnement et la saturation de la décharge d'Akouédo ne permettaient pas aux opérateurs intervenants de l'exploiter convenablement. En effet, il n'y avait vraiment plus de place pour recevoir ces tonnes d'ordures ménagères produites chaque jour par la population du District d'Abidjan. Selon les propos du superviseur du quai de déversement à la décharge en question, les opérateurs intervenants étaient donc obligés de tricher sur l'exploitation de cette décharge de façon à dissimuler les problèmes existants afin de toujours continuer à recevoir les déchets urbains. C'est pourquoi, Assi

${ }^{2}$ Pour rappel, au moment de la rédaction de notre article, la décharge publique d'Akouédo est définitivement fermée. Les déchets de la ville d'Abidjan sont transférés au centre d'enfouissement technique de Kossihouen situé à $40 \mathrm{~km}$ de la ville d'Abidjan. 
(2013) la qualifie de décharge hors norme à l'égard de la carence des moyens et techniques dont disposent les opérateurs et surtout de sa répercussion sur la qualité de l'environnement abidjanais.

Les résultats obtenus lors de l'étude sont aussi soutenus par ceux d'Amadou (2010), qui confirme les effets des carences du stade d'élimination finale des déchets de l'unique décharge de Mbeubeuss, ouverte en 1968 dans la ville Dakaroise. Son étude révèle la menace environnementale et sanitaire que représentent les décharges créées après l'ère coloniale dans les grandes villes africaines. De la même manière, les résultats d'Adjiri (2015), d'Onibokun et Kumuyi (2001) évaluent les moyens et techniques des opérateurs pour le traitement des déchets urbains sur les décharges non contrôlées. En effet, dans les grandes villes africaines tout comme dans le District d'Abidjan, les autorités responsables de l'unique décharge publique n'ont pas su anticiper ou du moins moderniser sa capacité d'accueil en tenant compte de la croissance démographique bien qu'ayant bénéficié des fortunes diverses en matière d'équipements et de construction d'infrastructures au sortir de l'indépendance. Enfin, cette carence a montré les limites de la gestion de la décharge d'Akouédo dans le temps, d'où la saturation de ce dépotoir qui se confond de plus en plus avec le village d'Akouédo.

\section{Conclusion}

La présente étude a permis de mettre en évidence les impacts dans l'exploitation de la décharge non contrôlée d'Akouédo, sur le cadre de vie et la santé de la population riveraine à l'égard des moyens et techniques dont disposent les opérateurs.

Les résultats obtenus nous permettent de conclure que les étapes de traitement et d'élimination des déchets urbains à la décharge d'Akouédo sont loin d'être écologiques et constituent une menace pour l'environnement et le bien-être de la population riveraine. Le dépôt d'ordures en question se confond de plus en plus avec les habitations de la population riveraine, dont le cadre de vie et la santé se voient très impactés à cause de l'exposition à des odeurs nauséabondes, à des maladies et des symptômes associés à la composition microbiologique des déchets urbains (diarrhée aigüe, fièvre typhoïde, infections pulmonaires, etc.) émanant de ladite décharge et à la présence de moustiques et rongeurs, insectes et mouches.

En définitive, les faiblesses de fonctionnement et d'exploitation de la décharge d'Akouédo ressorties dans notre étude pourraient servir à d'autres centres d'enfouissement technique en Afrique pour le renforcement de leur dispositif afin qu'ils ne subissent pas le même sort.

Ce sujet devrait aussi être examiné à fond si nous voulons léguer aux générations futures un environnement propre et préservé dans le District d'Abidjan. 


\section{References:}

1. Adjiri, O., Mafou, K., \& Konan, P. (2015). Impact de la décharge d'Akouédo (Abidjan - Côte d'Ivoire) sur les populations: étude socioéconomique et environnementale. International Journal of Innovation and Applied Studies, vol. 13, no. 4, pp. 979-989.

2. Amadou, B. (2010). Les déchets solides à Dakar. Environnement, sociétés et gestion urbaine, Geography. Université Michel de Montaigne - Bordeaux III, 793p, 2010.

3. Assi, G. (2013). Akouédo une décharge hors normes à Abidjan. In Yapi, A. Variations ivoiriennes : Revue Des Hautes Terres n4, vol.1. Yaoundé : IRESMA Editions, pp.11-22.

4. Ble, O., Kouadio, E., \& Bongoua, J. (Eds.) (2014). Evaluation de la qualité physico-chimique des eaux souterraines dans la zone d'Akouédo (Sud de la Côte d'Ivoire). International Journal of Innovation and Applied Studies, vol. 7, no. 3, pp. 1178-1190.

5. Hebette, A. (1996). Guide pratique de la gestion des déchets solides urbains en Afrique subsaharienne. Luxembourg: Lux-Development, $151 \mathrm{p}$.

6. Issihaka, A., Belhadj, S., \& El Bari, H. (Eds.). Contribution à l'amélioration de la gestion des déchets ménagers aux Comores. International Journal of Innovation and Applied Studies, vol. 12, no. 4, pp. 786-800.

7. Lamchouri, F., Zalaghi, A., \& Toufik, H. (2018). Utilisation d'un système de management intégré, Qualité-Sécurité-Environnement, pour la réhabilitation de la décharge publique non contrôlée de la ville de Taza (Maroc). International Journal of Innovation and Applied Studies, vol.23, no. 2, pp. 180-191.

8. Lamchouri, F., Zalaghi, A., \& Toufik, H. (Eds.) (2018). Traitement par le procédé SBR (Sequencing Batch Reactor) des lixiviats de la décharge publique non contrôlée de la ville de Taza (Maroc).International Journal of Innovation and Applied Studies, vol.23, no. 3, pp. 299-309.

9. Onibokun, A., \& Kumuyi, A. (2001). La gouvernance et la gestion des déchets en Afrique. In Onibokun, A.G. Gestion des déchets urbains : des solutions pour l'Afrique. Paris: CRDI-Karthala, pp. 5-11.

10. Sanogo, M. (2015). Les enjeux et défis d'une urbanisation durable pour la Côte d'Ivoire, In: Kouadio, R., Cissé, B., \& Grégoire, L. (Eds.), Développement durable et Emergence de l'Afrique, Grandvaux, pp. 575-584 